\section{Point spread function by random phase reference in collinear holographic storage}

Yeh-Wei Yu, Chih-Yuan Cheng, Shu-Ching Hsieh, Tun-Chien Teng, and Ching-Cherng Sun, FELLOW SPIE National Central University Department of Optics and Photonics Chung-Li 320, Taiwan

E-mail: ccsun@dop.ncu.edu.tw

Abstract. We use a paraxial approximating solution to calculate the point spread function of the collinear holographic storage system and show that the point spread function can be dramatically enhanced by the reference pattern with random binary phase modulation or random phase modulation. (๑) 2009 Society of Photo-Optical Instrumentation Engineers. [DOI: 10.1117/1.3080725]

Subject terms: volume holographic; storage; point spread function; collinear; coaxial; random phase; random binary phase.

Paper 080825LR received Oct. 18, 2008; revised manuscript received Dec. 9, 2008; accepted for publication Dec. 22, 2008; published online Feb. 10, 2009.

Collinear holographic storage (CHS) was proposed by Horimai ${ }^{1}$ in 2003 . This system intrinsically has many advantages including compatible with existing disk storage systems $^{2-4}$ such as CDs and DVDs. In addition, uniform shift selectivity in both radial and tangential directions and a fairly large wavelength shift and tilt tolerance are observed. ${ }^{5}$ However, the point spread function (PSF) of the proposed CHS is too wide spreading and as a result the diffracted signal is blurred, so the storage capacity in one page is limited. This restriction was not removed until the radial line reference pattern was proposed ${ }^{3}$ in 2005 . One of the disadvantages of the radial line reference is that the energy is wasted due to the low transmitting ratio in area in the reference pattern. The dc peak with extremely high energy in the focal point also causes saturation of the material and decreases the quality of the hologram. Introducing defocus of the object lens is a way to blur the dc peak to avoid this overexposure effect. However, for a CHS system, the reference beam and the signal beam interfere only around the focal plane. Defocus will decrease the interference region inside the media and inevitably decrease the effective dynamic range of the medium. A different approach is to use random binary phase (RBP) mask or random phase (RP) mask to make the spatial spectrum wider and suppress the dc component in the Fourier plane. ${ }^{6,7}$ This method will not decrease the interference region inside the media and is more suitable for solving the problem of dc peak. In this paper, we calculate the PSF of the collinear holographic storage system when reference pattern are RBP modulation and RP modulation. The simulation results show that with proper arrangement both the RBP mask and the RP mask can achieve extremely sharp PSFs.

Figure 1 shows a schematic diagram of the storage sys-

0091-3286/2009/\$25.00 @ 2009 SPIE (a)

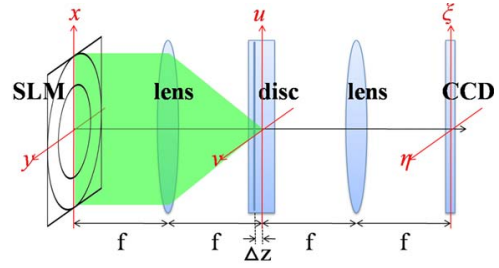

(b)



Fig. 1 Transmission model of the collinear algorithm for (a) a writing process and (b) a reading process.

tem for theoretical modeling. In the model, the mirror on the back surface of the holographic disk in the real system is replaced by a double-thickness disk in a modeled transmission algorithm to replace the reflection algorithm in the real system. In previous research, we showed that the PSF for the collinear system can be written ${ }^{8}$ as

$$
\begin{aligned}
\operatorname{PSF}(\xi, \eta)= & \frac{\exp (j k 4 f)}{(\lambda f)^{2}} \int_{-T}^{T} \exp \left[\frac{j \pi \Delta z}{\lambda f^{2}}\left(\xi^{2}+\eta^{2}\right)\right][\delta(\xi, \eta) \\
& \otimes \operatorname{psfz}(\xi, \eta, \Delta z)] \mathrm{d} \Delta z
\end{aligned}
$$

where $\xi$ and $\eta$ are the lateral coordinates, $\Delta z$ is the longitudinal deviation from the center of the disk, $T$ is the thickness of the disk, $k$ is the wave number, $\lambda$ is the effective wavelength, $f$ is the effective focal length of the Fourier transform lens, $\delta(\xi, \eta)$ is a delta function that stands for the point input of the signal, and psfz denotes the PSF by the diffraction from each layer of the hologram and is expressed as

$$
\begin{aligned}
\operatorname{psfz}= & \left\{U_{p}(-\xi,-\eta) \exp \left[-j \frac{\pi \Delta z}{\lambda f^{2}}\left(\xi^{2}+\eta^{2}\right)\right]\right\} \\
& \otimes\left\{U_{r}^{*}(\xi, \eta) \exp \left[j \frac{\pi \Delta z}{\lambda f^{2}}\left(\xi^{2}+\eta^{2}\right)\right]\right\},
\end{aligned}
$$

where $U_{p}(\xi, \eta)$ and $U_{r}(\xi, \eta)$ are the reading and the reference patterns, respectively. Since the reading pattern is always the same as the reference pattern, the convolution in Eq. (2) becomes the autocorrelation of the reference pattern with a phase term (or the reading pattern). Then from Eq. (1) we find that the PSF is a result of the integration of the psfz multiplied by a quadratic phase term across the whole volume of the hologram. Consequently, a welldesigned reference pattern could reduce the PSF.

We use the three different reference patterns shown in Fig. 2, where Fig. 2(a) is the radial pattern with line width of $13.68 \mu \mathrm{m}$, Fig. 2(b) is the RBP mask with the modulation pitch of $13.68 \times 13.68 \mu \mathrm{m}$, and Fig. $2(\mathrm{c})$ is the RP mask with the modulation pitch of $13.68 \times 13.68 \mu \mathrm{m}$. The simulation result of the intensity distribution in the $x$ direction for the corresponding reference patterns is shown in 


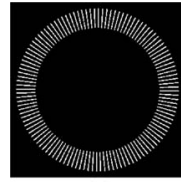

(a)

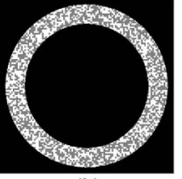

(b)

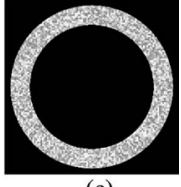

(c)
Fig. 2 Three kinds of reference patterns.

Fig. 3, where $T=0.6 \mathrm{~mm}$, and the size of the spatial light modulator $(\mathrm{SLM})$ is $4.4 \times 4.4 \mathrm{~mm}$. The pitch between each pixel in the SLM is $13.68 \mu \mathrm{m}$. In the calculation, the effective focal length is set to $7.5 \mathrm{~mm}$ and the effective wavelength is $532 \mathrm{~nm} / 1.5$ to simplify the calculation of the refraction on the boundary between the air and the holographic disk. We find that the first zero point of the PSF is the same in all three modulation cases. However, the sidelobe of the PSF can be dramatically reduced by RBP and RP modulation.

Then we change the modulation pitch from 13.68 to $1.05 \mu \mathrm{m}$ for both RBP and RP modulation. Figure 4 shows the simulation result. We find that the simulation result for RBP modulation is similar to that for RP modulation. When the modulation pitch is $1.05,2.10,3.15$, and $13.68 \mu \mathrm{m}$, the first zero point of the PSF locates at 1.05, $2.10,3.15$, and $13.68 \mu \mathrm{m}$, respectively. This shows that the PSF can be improved by just narrowing the modulation pitch. The width of the PSF can be shortened to $2.1 \mu \mathrm{m}$ when the modulation pitch is $1.05 \mu \mathrm{m}$.

In summary, we proposed a theoretical analysis for calculating the PSF of a collinear holographic storage system when the reference pattern is RBP or RP modulation. The

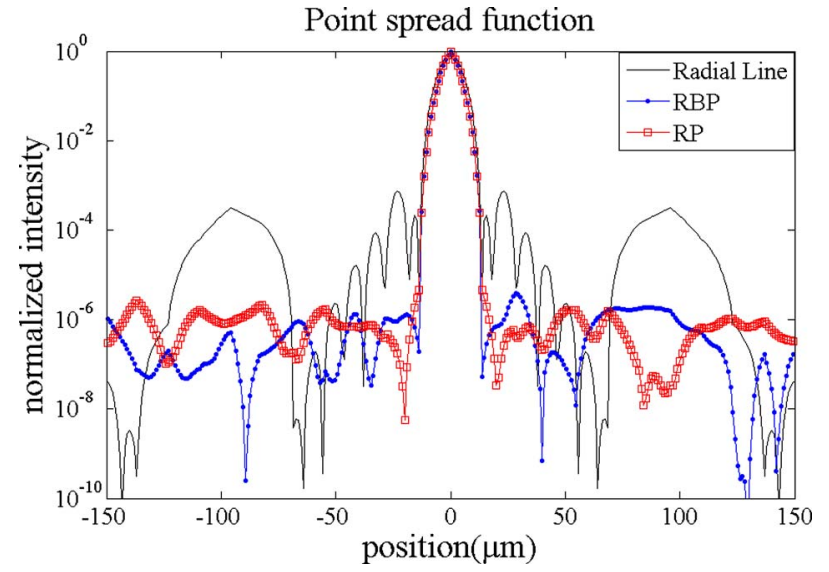

Fig. 3 PSF with different kinds of modulation.

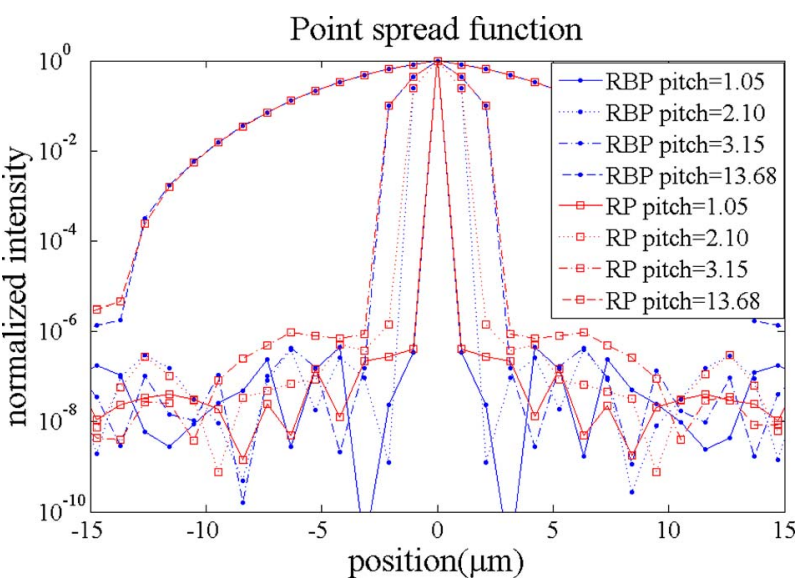

Fig. 4 PSF for RBP and RP modulation with different pitches.

simulation results show that both RBP and RP modulation can be used to improve the PSF, and their performances are similar. In addition, we can shorten the width of the PSF to $2.1 \mu \mathrm{m}$ when the modulation pitch of the RBP mask or the $\mathrm{RP}$ mask is as small as $1 \mu \mathrm{m}$.

\section{Acknowledgments}

This study was sponsored by the Ministry of Economic Affairs of R.O.C. under Grant No. 95-EC-17-A-07-S1-011 and the National Science Council under Grant No. NSC 96-2221-E-008-031.

\section{References}

1. H. Horimai, "Collinear holography," in Proc. 5th Pacific Rim Conf. on Lasers and Electro-Optics, Vol. 1, pp. 376-377, Taiwan (2003).

2. H. Horimai and X. Tan, "Advanced collinear holography," Opt. Rev. 12, 90-92 (2005).

3. H. Horimai, X. D. Tan, and J. Li, "Collinear holography," Appl. Opt. 44, 2575-2579 (2005).

4. K. Tanaka, H. Mori, M. Hara, K. Hirooka, A. Fukumoto, and K. Watanabe, "High density recording of $270 \mathrm{Gbits} /$ inch $^{2}$ in a coaxial holographic storage system," in Technical Digest of ISOM 2007 (2007).

5. H. Horimai and X. Tan, "Holographic versatile disc system," Proc. SPIE 5939, 593901 (2005).

6. K. Tanaka, M. Hara, K. Tokuyama, K. Hirooka, K. Ishioka, A Fukumoto, and K. Watanabe, "Improved performance in coaxial holographic data recording," Opt. Express 15, 16196-16209 (2007).

7. C. B. Burckhardt, "Use of a random phase mask for the recording Fourier transform holograms of data masks," Appl. Opt. 9, 695-700 (1970).

8. C. C. Sun, Y. W. Yu, S. C. Hsieh, T. C. Teng, and M. F. Tsai "Point spread function of a collinear holographic storage system," Opt. Express 15, 18111-18118 (2007) 